\title{
ENTROPY GENERATION RATE IN A MICROSCALE THIN FILM
}

\author{
Saad Bin Mansoor ${ }^{1, *}$
}

\begin{abstract}
This paper presents a new formulation of the rate of entropy generation in thin films whose thickness is of the order of the mean-free-path or less. In this relation, an expression for the gradient of the equivalent equilibrium temperature is proposed that is a function of the gradient of the phonon intensity at any point inside the thin film. It is shown that the proposed expression reduces to the familiar gradient of the thermodynamic temperature in the diffusive limit. Furthermore, the new formulation is used to compute the entropy generation rate for the case of steady-state, one-dimensional heat transfer in a thin film by first solving the Equation of Phonon Radiative Transfer to determine the phonon intensity. These computations are performed both for the silicon and the diamond thin films, for a range of Knudsen numbers starting from the diffusive limit up until the ballistic limit. It is found that the entropy generation rate attains a peak value at $\mathrm{Kn}=0.7$ and decreases for other Knudsen numbers when non-equilibrium transport is adopted in the analysis. However, rate of entropy generation increases almost linearly for the equilibrium heating situation. This is true for both the silicon and the diamond thin films.
\end{abstract}

\section{Keywords: Phonon Transport, Non-equilibrium Heating, Thin Films, Entropy Generation Rate}

\section{INTRODUCTION}

Energy transport across the thin film films involves with non-equilibrium processes, which can be described through the wave nature of transfer characteristics. In general, phonons are the waves, which govern the energy transfer inside the thin films when the film is thermally disturbed from the edges. One of the key factors effecting the transport characteristics is the ratio of phonon mean free path of the film over the film size, which is described through the Knudsen number $(\mathrm{Kn})$. For the condition when $\mathrm{Kn} \geq 1$, the continuum approach describing the energy transport across the film fails the describe the phenomena correctly because of the ballistic contribution of large wavelength phonons. In this case, the ballistic phonons do not undergo scattering in the film, they rather jump across the film edges while not seeing the film resistance to the thermal transport. Describing such transfer processes incorporating the classical approaches, such as Fourier model, fails to predict the correct temperature rise or equilibrium phonon intensity distribution in the film. One of the approached for the non-equilibrium transport process is to incorporate the Boltzmann Transport Equation describing the phonon intensity distribution in the film. The Boltzmann Equation incorporates the wave nature of the transport characteristics and provides the relation for the phonon intensity distribution inside the film. However, the solution of the Boltzmann equation is rather complicated and simplification such as Matthiessen rule describing the relaxation time reduces the analytical complexity and numerical efforts. In this case, The Matthiessen rule is an empirical rule which states that the total resistivity of a crystalline metallic specimen is the sum of the resistivity due to thermal agitation of the metal ions of the lattice and the resistivity due to the presence of imperfections in the crystal. The lattice imperfections and thermal resistance of the film initiate the entropy generation rate in the film. The formulation of the entropy generation rate relies on the phonon intensity distribution inside the film for those phonons that undergo scattering [1]. However, various approaches can possibly be considered to formulate the entropy generation rate within the film such as the one purposed in the present study.

On the other hand, it is well-known that conduction heat transfer in microscale devices is not correctly described by the Fourier law of heat diffusion [1]. Instead, one has to model the heat conduction through the phenomenon of phonon transport. Considerable research has been carried out in this area. One of the approaches is to model the thermal energy transfer through phonons by means of the Boltzmann Transport Equation (BTE). A modified version of the BTE was derived in [1] and is termed as the Equation of Phonon Radiative Transfer or EPRT. This equation is similar to the Radiative Transfer Equation (RTE) as used in the theory of radiation heat transfer. Solution of the EPRT not only provides the heat flux inside a microscale thin film but also the equivalent equilibrium temperature, which in the diffusive limit reduces to the usual thermodynamic temperature. However, the EPRT is limited to small temperature differences between the boundaries. The topic of entropy generation rate during radiation heat transfer in a participating media has recently seen increased interest. It was originally treated much earlier by Max Planck [2]. Recently, various researchers have investigated this topic [3, 4, 5]. Since the mathematical description of conduction heat transfer in the ballistic- 
diffusive limit is similar to radiative heat transfer in a participating media, therefore, the entropy generation rate formalism of the former is similar to that of the later. This point of view is explored in a paper by Bright and Zhang [6]. They formulated the expression for the volumetric entropy generation rate in a one-dimensional, thin film and then used it to compute the entropy generation rate for various values of the Knudsen numbers. Ali and Yilbas [7] has also explored the phenomenon of entropy generation in thin films with thickness of the order of the mean free path of the phonon carriers. They studied the effect of changing the film thickness on the entropy generation rate.

In the present study an attempt is made to compute the entropy generation inside a microscale thin film by modifying the entropy generation rate formula that is applicable in the diffusive limit. The modification is carried out in a manner such that in the limit when the film thickness is large and conduction heat transfer reaches the diffusive regime, the proposed expression for the entropy generation rate also approaches the classical expression.

\section{MATHEMATICAL METHOD} is given as [8],

The entropy generation rate at any point in a solid body that is under local thermodynamic equilibrium

$$
\dot{S}_{g e n}^{\prime \prime \prime}=-\frac{\mathbf{q}^{\prime \prime} \cdot \nabla T}{T^{2}}
$$

In Eq. (1) $\mathbf{q}^{\prime \prime}$ is the heat flux vector at a point and $\nabla T$ is the gradient of the thermodynamic temperature at that point. Eq. (1) is valid for steady-state and transient problems with or without a volumetric heat source/sink. When the device size is of the order of the phonon mean-free-path or smaller, then the dielectric film is under non-equilibrium conditions and therefore one cannot define the thermodynamic temperature at any point inside the device. In its place, one can define an equivalent equilibrium temperature. It can be shown that in the limit of a larger device size, the equivalent equilibrium temperature reduces to the usual thermodynamic temperature. However, this does not imply that the gradient of the thermodynamic temperature can also be approximated by the gradient of the equivalent equilibrium temperature. In the analysis below, we propose an expression for the gradient of the thermodynamic temperature that can also be used in nonequilibrium situations. Consider the following expression for the temperature gradient,

$$
\nabla T=\frac{3}{C \nu} \int_{0}^{2 \pi} \int_{0}^{\pi} \boldsymbol{\Omega}[\nabla \cdot(\boldsymbol{\Omega} I)] \sin \theta d \theta d \phi
$$

In Eq. (2), $\nabla T$ is taken as a single entity when using it in the thermodynamic non-equilibrium regime and it represents the gradient of the equivalent equilibrium temperature. In the diffusive limit $\nabla T$ represents the usual gradient of the thermodynamic temperature as shown later. $C$ is the volumetric heat capacity in $J /\left(m^{3} K\right), v$ is the phonon group velocity in $m / s, \boldsymbol{\Omega}$ is the unit vector in a generic direction, $I$ is the phonon intensity, $\theta$ is the polar angle and $\phi$ is the azimuthal angle. For the radiative transfer phenomenon it can be shown that Eq. (2) can also be written as,

$$
\nabla T=\frac{3}{C \nu} \int_{0}^{2 \pi} \int_{0}^{\pi} \boldsymbol{\Omega}(\boldsymbol{\Omega} \cdot \nabla I) \sin \theta d \theta d \phi
$$

or,

$$
\nabla T=\frac{3}{C v} \int_{0}^{2 \pi} \int_{0}^{\pi}(\mathbf{\Omega} \mathbf{\Omega}) \cdot(\nabla I) \sin \theta d \theta d \phi
$$

In Eq. (3b), $\boldsymbol{\Omega} \boldsymbol{\Omega}$ is the direct product of two vectors and is termed as a dyad. In the limit when the Knudsen number tends to zero and heat conduction is through diffusion only, the phonon intensity $I \rightarrow I^{o}$, where $I^{o}$ is the equilibrium intensity and is independent of direction. It is defined as, 


$$
I^{o}=\frac{C v}{4 \pi} T
$$

In any coordinate system whether Cartesian, orthogonal or non-orthogonal, the unit vector $\boldsymbol{\Omega}$ at any point can always be written as a linear combination of three mutually orthogonal unit vectors $\hat{\mathbf{e}}_{1}, \hat{\mathbf{e}}_{2}, \hat{\mathbf{e}}_{3}$, i.e., $\boldsymbol{\Omega}=\cos \theta \hat{\mathbf{e}}_{1}+\sin \theta \cos \phi \hat{\mathbf{e}}_{2}+\sin \theta \sin \phi \hat{\mathbf{e}}_{3} \quad$ [9], where $\theta$ and $\phi$ are the polar and azimuthal angles respectively. We now substitute Eq. (4) in to Eq. (3b) to simulate the diffusive limit. Using the expression for $\boldsymbol{\Omega}$ and simplifying we get,

$$
\nabla T=\frac{3}{\not V v} \frac{\not \ell}{4 \pi}\left(\int_{0}^{2 \pi} \int_{0}^{\pi} \boldsymbol{\Omega} \boldsymbol{\Omega} \sin \theta d \theta d \phi\right) \cdot \nabla T=\frac{3 /}{A \pi} \frac{4 \pi /}{3}\left[\begin{array}{lll}
1 & 0 & 0 \\
0 & 1 & 0 \\
0 & 0 & 1
\end{array}\right] \cdot \nabla T=\nabla T
$$

Hence, in the thermodynamic equilibrium limit the proposed expression reduces to the gradient of the thermodynamic temperature. Therefore, it is expected that in the non-equilibrium regime Eq. (3) is the correct substitute for the temperature gradient. Returning back to the entropy generation rate we recall that,

$$
\mathbf{q}^{\prime \prime}=\int_{0}^{2 \pi} \int_{0}^{\pi} \mathbf{\Omega} I \sin \theta d \theta d \phi \quad\left(W / m^{2}\right)
$$

In Eq. (5), q" is the heat flux vector. Substituting Eq. (3) and Eq. (5) in to Eq. (1) we get the expression for the local entropy generation rate,

$$
\dot{S}_{g e n}^{\prime \prime \prime}=-\frac{3}{C \nu T^{2}}\left(\int_{0}^{2 \pi} \int_{0}^{\pi} \boldsymbol{\Omega} I \sin \theta d \theta d \phi\right) \cdot\left(\int_{0}^{2 \pi} \int_{0}^{\pi} \boldsymbol{\Omega}(\boldsymbol{\Omega} \cdot \nabla I) \sin \theta d \theta d \phi\right)\left(\frac{W}{m^{3} K}\right)
$$

In Eq. (6), the equivalent equilibrium temperature is defined as,

$$
T=\frac{1}{C \nu} \int_{0}^{2 \pi} \int_{0}^{\pi} I \sin \theta d \theta d \phi
$$
the volume.

The total entropy generation rate can be obtained by integrating the local entropy generation rate over

$$
\dot{S}_{g e n}=-\frac{3}{C \nu} \iiint_{\forall} \frac{1}{T^{2}}\left(\int_{0}^{2 \pi} \int_{0}^{\pi} \boldsymbol{\Omega} I \sin \theta d \theta d \phi\right) \cdot\left(\int_{0}^{2 \pi} \int_{0}^{\pi} \boldsymbol{\Omega}(\boldsymbol{\Omega} \cdot \nabla I) \sin \theta d \theta d \phi\right) d^{3} \forall \quad(W / K)
$$

Eq. (8) can be used to calculate the total entropy generation rate in the dielectric thin film. In the present study Eq. (8) is used to calculate the entropy generation rate in a thin dielectric film. The problem is onedimensional, steady-state and frequency dependent. Mathematically, the problem is formulated as,

$$
\mu \frac{\partial I}{\partial x}=\frac{\int_{-1}^{1} I d \mu-I}{\Lambda}
$$

where, $I=I(x, \mu), \mu=\cos \theta$ and $\Lambda$ is the phonon mean-free-path . The boundary conditions are,

$$
I(x=0,0 \leq \mu \leq 1)=\frac{C v T_{\text {left }}}{4 \pi}, \text { where } T_{\text {left }}=301 \mathrm{~K}
$$




$$
I(x=L,-1 \leq \mu \leq 0)=\frac{C v T_{\text {right }}}{4 \pi}, \text { where } T_{\text {right }}=300 \mathrm{~K}
$$

\section{NUMERICAL SOLUTION}

Figure (1) shows the schematic view of the thin film and left and right edges temperature boundary conditions. The Equation of Phonon Radiative Transfer in the silicon film with the associated initial and boundary conditions is solved numerically using the finite-difference method. This is an integro-differential equation and may be solved using an iterative technique. Consider the steady-state, frequency independent, EPRT in the silicon film:

$$
\mu \frac{\partial I}{\partial x}=\frac{\int_{-1}^{1} I d \mu-I}{\Lambda}
$$

The variable $\mu=\cos \theta$ vary between $-1 \leq \mu \leq 1$. To facilitate the solution of the EPRT it is customary to break the intensity into parts. When $-1 \leq \mu \leq 0, I=I^{-}(x, \mu)$ and when $0 \leq \mu \leq 1$, $I=I^{+}(x, \mu)$. Therefore, we may write separate equations for the two intensities. These are given below:

$$
\mu \frac{\partial I^{-}}{\partial x}=\frac{\int_{-1}^{0} I^{-} d \mu+\int_{0}^{1} I^{+} d \mu-I^{-}}{\Lambda} \quad-1 \leq \mu \leq 0
$$

and,

$$
\mu \frac{\partial I^{+}}{\partial x}=\frac{\int_{-1}^{0} I^{-} d \mu+\int_{0}^{1} I^{+} d \mu-I^{+}}{\Lambda} \quad 0 \leq \mu \leq 1
$$

To solve the above equations, the discrete ordinates method is used for the discretisation of the angular term. The partial derivative with respect to the coordinate variable $x$ is descritised using a forward-difference for the first equation in which $-1 \leq \mu \leq 0$ and is descritised using a backward-difference for the second equation in which $0 \leq \mu \leq 1$. This procedure is important to obtain stable discretisations. The integrals are calculated by first discretising the cosine of the polar angle $\mu$ into 40 divisions and then using the Simpson's rule to evaluate the integrals numerically for each time-step and each coordinate location. It is important to keep in mind that the intensities $\mathrm{I}^{-}$and $\mathrm{I}^{+}$are two separate variables and each needs a single boundary condition. For the intensity $I^{-}$, the boundary condition comes from the film right edge temperature condition. For the intensity $I^{+}$, the boundary condition comes from the film left edge temperature condition. Table 1 gives the properties of silicon and diamond films used in the simulations.

Table 1. Thermo-physical properties of Silicon and Diamond

\begin{tabular}{|c|c|c|}
\hline Property & Silicon & Diamond \\
\hline Volumetric heat capacity, $\mathbf{C}, \mathbf{( J / \mathbf { m } ^ { \mathbf { 3 } } / \mathbf { K } )}$ & $1.653 \times 10^{6}$ & $1.815 \times 10^{6}$ \\
\hline Bulk thermal conductivity, $\mathbf{k}, \mathbf{( W / m} / \mathbf{K})$ & 157 & 3320 \\
\hline Phonon group velocity, $\mathbf{v}, \mathbf{( m / s})$ & 8430 & 12288 \\
\hline Phonon mean-free-path, $\boldsymbol{\Lambda},(\mathbf{n m})$ & 33.8 & 446.6 \\
\hline
\end{tabular}

\section{RESULTS AND DISCUSSION}

The expression relying on the phonon intensity distribution is incorporated to determine the entropy generation rate for the case of one-dimensional, steady-state, heat transfer in a thin film for various Knudsen 
numbers. Two different film materials are considered namely, Silicon and Diamond, in the simulations. The thermo-physical properties employed in the simulations are listed in Table 1.

The Knudsen number is varied within the range $0.01-10$. To accommodate the variation of the Knudsen number, the film thickness is kept variable. Hence, depending on the Knudsen number, the film thickness $L$ is calculated based on the formula $K n=\Lambda / L$. It should be noted that the continuum approach becomes effective for $K n \leq 0.1$. In addition, equivalent equilibrium temperature is introduced to quantify the equilibrium phonon intensity distribution in the thin film. Equivalent equilibrium temperature is associated with the average energy of all phonons around a local point when the phonon energies are redistributed adiabatically to an equilibrium state.

Figures (2a) and (2b) show normalized equivalent equilibrium temperature $\left(\frac{T-T_{\min }}{T_{\max }-T_{\min }}\right.$, where $T$ is equivalent equilibrium temperature, $T_{\min }$ is the low temperature at the edge, $T_{\max }$ is the maximum temperature at the high temperature edge of the film) with the normalized film thickness ( $x / L$, where $L$ is the film thickness) for silicon and diamond film materials, respectively. Temperature decays from high temperature edge towards the low temperature edge in thin film. However, temperature decay becomes almost linear for the $\mathrm{Kn} \leq 0.01$. In this case, equilibrium heating governs the transport process and Fourier heating model can be applicable describing the temperature distribution within the film. However, as the Kn increases, non-equilibrium takes place, which modifies the linear temperature decay to non-linear form. This situation is more pronounced as the film thickness reduces further (where Kn reduces further). In addition, temperature jump takes place at the film edges. The temperature jump is related to the boundary scattering of the emitted phonons from the edges. It should be noted that the phonons with various wavelength are emitted from the film edges and the emitted phonons suffer from scattering in the film while generating the film thermal resistance. Consequently, boundary scattering further reduces the emitted phonon intensity in the vicinity of the boundary as the film thickness reduces significantly. The ballistic phonons do not contribute to the scattering process and they jump from one edge to the other edge of the film when their wavelengths become larger than the film thickness. In addition, phonons emitted from the low temperature edge also contribute to the film resistance via scattering in the film. However, phonon intensity emitted from the low temperature edge remains low as compared to those emitted from the high temperature edge. Consequently, their contribution becomes relatively smaller than those of the emitted phonons from the high temperature edge. Consequently, low temperature edge of the film acts like a phonon sink while lowering the phonon intensity in the low temperature edge region. When comparing normalized equivalent equilibrium temperature for silicon and diamond thin films, both films remain similar response to temperature disturbance applied from both film edges.

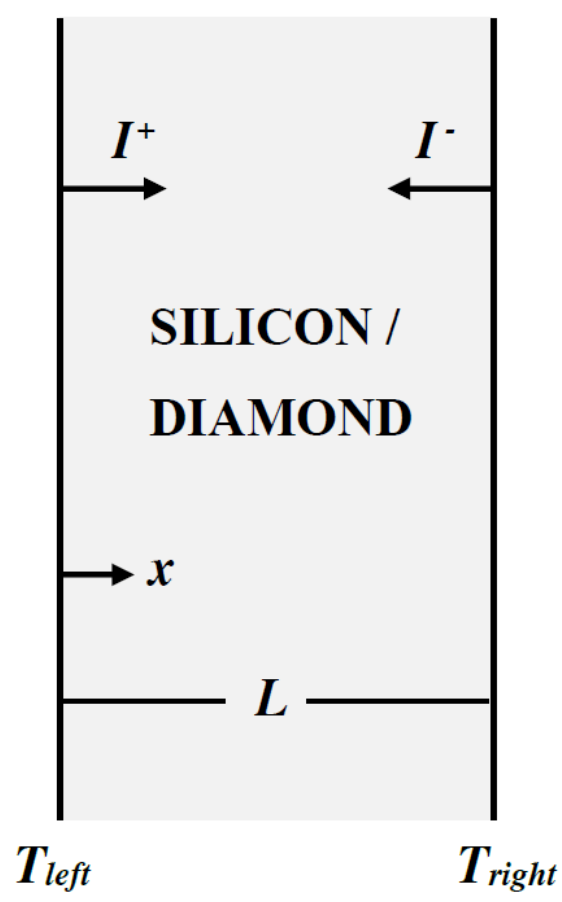

Figure 1. Schematic of the thin film geometry and boundary condition for EPRT solution 


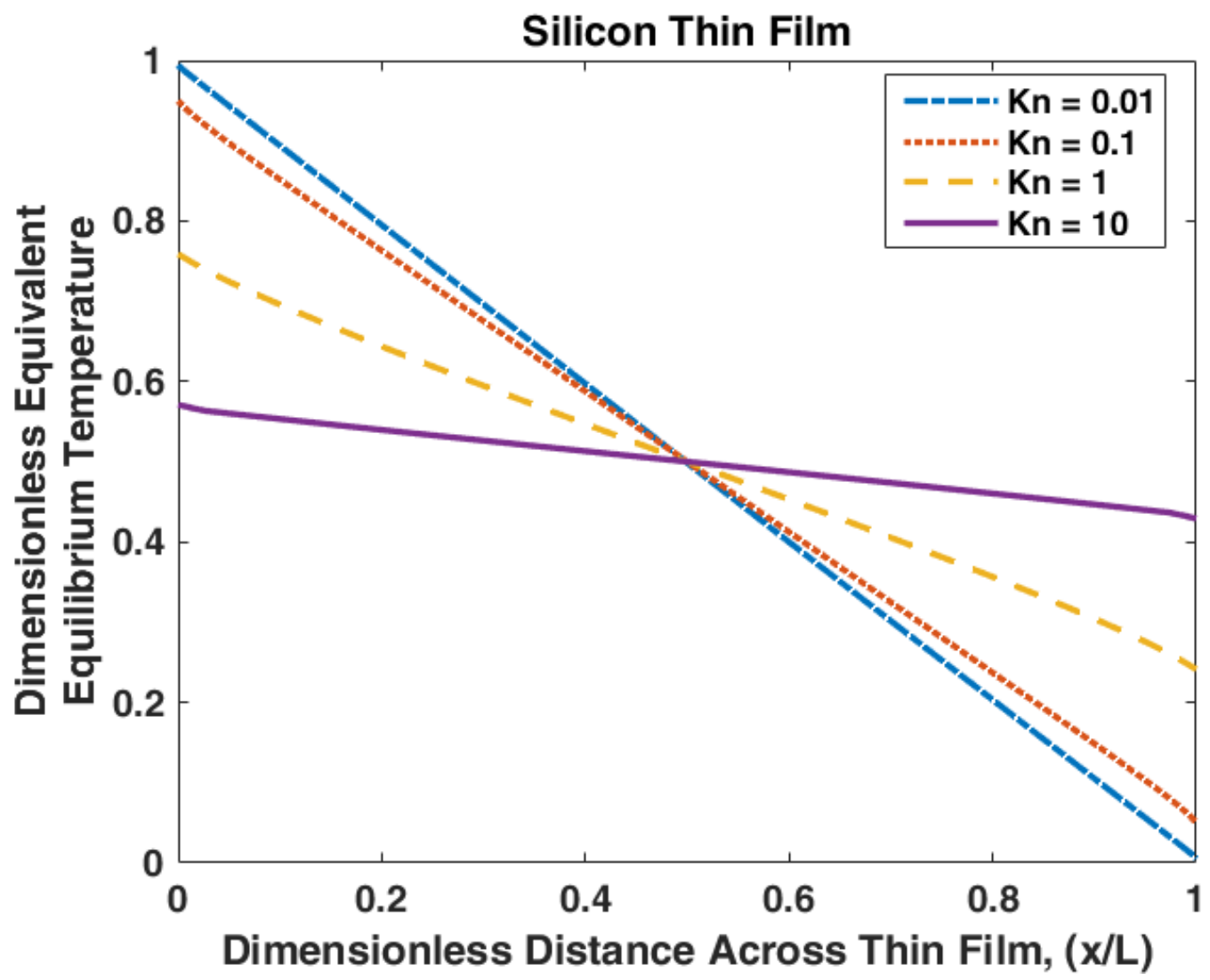

(a)

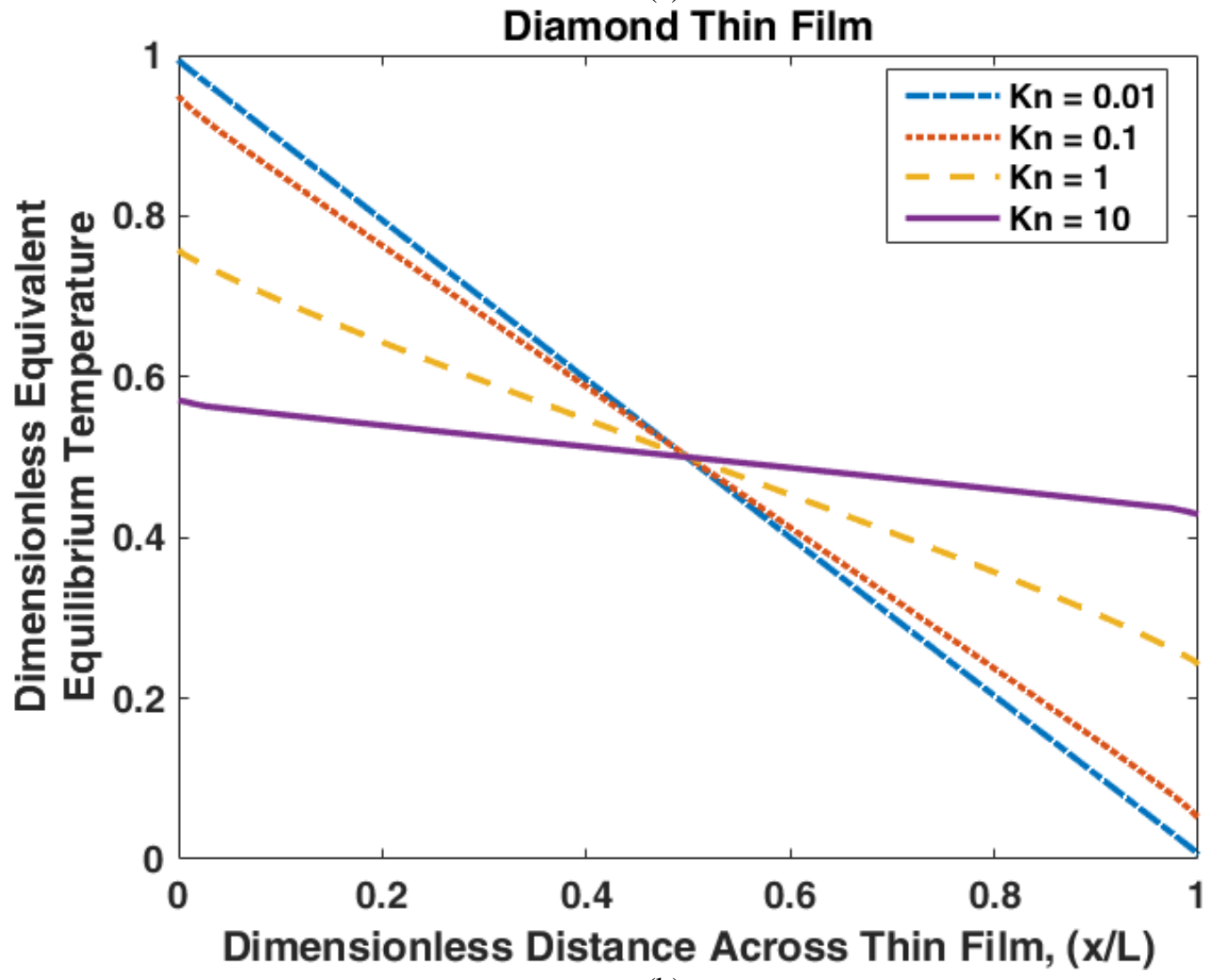

(b)

Figure 2. (a) Spatial distribution of the equivalent equilibrium temperature for various Knudsen numbers in Silicon thin film, (b) Spatial distribution of the equivalent equilibrium temperature for various Knudsen numbers in Diamond thin film 
Figure (3) shows the total entropy generation rate with the Knudsen number $(\mathrm{Kn}=\Lambda / L$, where $\Lambda$ is the phonon mean free path) for silicon and diamond films. The entropy generation rate obtained from the equilibrium analysis incorporating the Fourier heating is also presented for comparison. The rate of entropy generation remains almost identical for equilibrium (Fourier analysis) and non-equilibrium (Phonon Radiative Transport) heating situations for $\mathrm{Kn} \leq 0.1$. However, increasing Kn results in significant deviation of the entropy generation rate due to equilibrium and non-equilibrium considerations. Since the low values of Kn corresponds to the thick films, the ballistic phonon contribution to the energy transport becomes negligible. This, in turn, gives rise to the equilibrium heating situation. Therefore, equilibrium and non-equilibrium approaches converge in terms of the entropy generation rate. As the Kn increases, the film thickness increases. This in turn results in significant deviation of the entropy generation rate because of the non-equilibrium heating involved. The equilibrium consideration (Fourier analogy) results in steady increase of entropy generation rate regardless of the film thickness. However, in the case of non-equilibrium heating situation, the rate of entropy generation increases first and the rate decreases with further increasing $\mathrm{Kn}$. This behavior is attributed to the energy transport by the ballistic transport, which do not contribute to the film thermal resistance; consequently, the entropy generation rate due to film thermal resistance. Moreover, the entropy generation increases regardless of the film thickness and the send law thermodynamics is not violated. When comparing the entropy generation rate due to silicon and diamond films, entropy generation rate remains higher for the silicon film than that of the diamond film. This behavior is attributed to the phonon intensity distribution of the silicon and diamond thin films (figures (2a) \& (2b)) because of different properties in each film as given in table 1 .

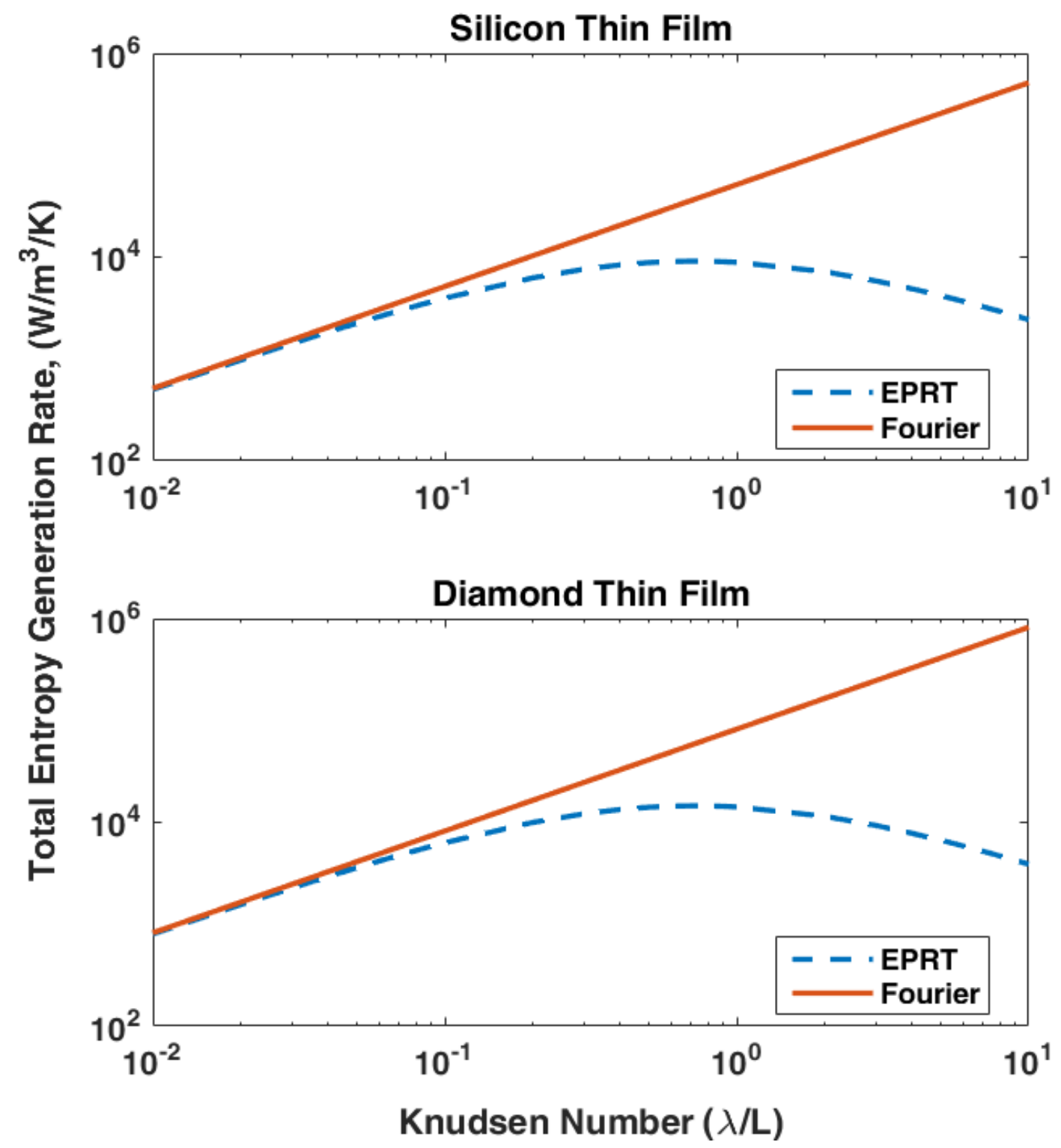

Figure 3. Variation of the total entropy generation rate with Knudsen number in thin films: (a) Silicon, (b) Diamond 


\section{CONCLUDING REMARKS}

Non-equilibrium energy transport in silicon and diamond thin films are considered for various Knudsen number and entropy generation rate is formulated incorporating the equilibrium phonon intensity distribution inside the film. The equation for phonon radiative transport is incorporated when formulating the phonon intensity distribution inside the film. Equivalent equilibrium temperature is introduced to quantify the equilibrium phonon intensity distribution in the film. Thin films are thermally disturbed from edges through introducing two different constant film edge temperatures ( $301 \mathrm{~K}$ and $300 \mathrm{~K}$ ). It is found that, as Knudsen number increases beyond unity, the ballistic phonon contribution to the energy transport becomes important. In this case, boundary scattering of phonons gives rise to temperature jump in the vicinity of the film edges. In addition, equivalent equilibrium temperature distribution across the film differs significantly from that of the equilibrium heating situation. Entropy generation rate resulted from the non-equilibrium consideration differs from that obtained from the equilibrium consideration. Entropy generation rate increases with Knudsen number steady for the case of equilibrium heating situation. However, the rate of entropy generation slows for the nonequilibrium case, which is more pronounced for increasing Knudsen number beyond unity. This behavior is attributed to the ballistic phonon contribution to the energy transport across the film edges. Entropy generation rate attains higher values for silicon thin film than the diamond film because of the film properties.

This study provides insight into the phonon transport inside the film for various Knudsen number and provides useful information on the entropy generation rate for equilibrium and non-equilibrium heating situations.

\section{ACKNOWLEDGMENTS}

The author acknowledge the support of King Fahd University of Petroleum and Minerals, Dhahran, Saudi Arabia, for this work.

\section{NOMENCLATURE}

$\begin{array}{ll}C & \text { Volumetric heat capacity }\left(\mathrm{J} / \mathrm{m}^{3} / \mathrm{K}\right) \\ \hat{\mathbf{e}}_{1}, \hat{\mathbf{e}}_{2}, \hat{\mathbf{e}}_{3} & \text { Unit basis vectors } \\ I & \text { Phonon Intensity }\left(\mathrm{W} / \mathrm{m}^{2}\right) \\ L & \text { Film thickness }(\mathrm{m}) \\ \mathbf{q}^{\prime \prime} & \text { Heat Flux Vector }\left(\mathrm{W} / \mathrm{m}^{2}\right) \\ \dot{S}_{g e n}^{\prime \prime \prime} & \text { Volumetric entropy generation rate }\left(\mathrm{W} / \mathrm{m}^{3} / \mathrm{K}\right) \\ t & \text { Time (s) } \\ T & \text { Equivalent equilibrium temperature }(\mathrm{K}) ; \text { Gray-body temperature (K); Thermodynamic } \\ v & \text { temperature }(\mathrm{K}) \\ x & \text { Phonon group velocity }(\mathrm{m} / \mathrm{s}) \\ \Lambda & \text { Cartesian coordinate }(\mathrm{m}) \\ \mu & \text { Phonon mean-free-path }(\mathrm{m}) \\ \theta & \text { Cosine of the polar angle } \\ \phi & \text { Polar angle } \\ \mathbf{\Omega} & \text { Azimuthal angle } \\ & \text { Unit vector }\end{array}$

\section{REFERENCES}

[1] Majumdar, A. S. M. E. (1993). Microscale heat conduction in dielectric thin films. Journal of Heat Transfer, 115(1), 7-16.

[2] Planck, M. (1959). The Theory of Heat Radiation. Dover: New York.

[3] Caldas, M., Semiao, V. (2005). Entropy generation through radiative transfer in participating media: analysis and numerical computation. Journal of Quantitative Spectroscopy and Radiative Transfer, 96(3-4), 423-437.

[4] Liu, L. H., Chu, S. X. (2006). On the entropy generation formula of radiation heat transfer processes. Journal of heat transfer, 128(5), 504-506.

[5] Zhang, Z. M., Basu, S. (2007). Entropy flow and generation in radiative transfer between surfaces. International journal of heat and mass transfer, 50(3-4), 702-712.

[6] Bright, T. J., Zhang, Z. M. (2010). Entropy generation in thin films evaluated from phonon radiative transport. Journal of Heat Transfer, 132(10), 101301.

[7] Ali, H., Yilbas, B. S. (2014). Entropy generation in silicon thin film: Influence of film thickness on entropy generation rate. Journal of Non-Equilibrium Thermodynamics, 39(3), 147-158. 
Journal of Thermal Engineering, Technical Note, Vol. 5, No. 5, pp. 405-413, October, 2019

[8] Aziz, A., Khan, W. A. (2012). Entropy generation in an asymmetrically cooled slab with temperaturedependent internal heat generation. Heat Transfer-Asian Research, 41(3), 260-271.

[9] Yilbas, B. S., Mansoor, S. B., Ali, H. (2017). Heat Transport in Micro-and Nanoscale Thin Films. Elsevier. 